HU-EP-07/12

arXiv:0704.0929

\title{
Noncommutative Electromagnetism As A Large $N$ Gauge Theory
}

\author{
Hyun Seok Yang* \\ Institut für Physik, Humboldt Universität zu Berlin \\ Newtonstraße 15, D-12489 Berlin, Germany
}

\begin{abstract}
We map noncommutative (NC) $U(1)$ gauge theory on $\mathbf{R}_{C}^{d} \times \mathbf{R}_{N C}^{2 n}$ to $U(N \rightarrow \infty)$ Yang-Mills theory on $\mathbb{R}_{C}^{d}$, where $\mathbf{R}_{C}^{d}$ is a $d$-dimensional commutative spacetime while $\mathbb{R}_{N C}^{2 n}$ is a $2 n$-dimensional NC space. The resulting $U(N)$ Yang-Mills theory on $\mathbf{R}_{C}^{d}$ is equivalent to that obtained by the dimensional reduction of $(d+2 n)$-dimensional $U(N)$ Yang-Mills theory onto $\mathbf{R}_{C}^{d}$. We show that the gauge-Higgs system $\left(A_{\mu}, \Phi^{a}\right)$ in the $U(N \rightarrow \infty)$ Yang-Mills theory on $\mathbb{R}_{C}^{d}$ leads to an emergent geometry in the $(d+2 n)$-dimensional spacetime whose metric was determined by Ward a long time ago. In particular, the 10-dimensional gravity for $d=4$ and $n=3$ corresponds to the emergent geometry arising from the 4-dimensional $\mathcal{N}=4$ vector multiplet in the AdS/CFT duality. We further elucidate the emergent gravity by showing that the gauge-Higgs system $\left(A_{\mu}, \Phi^{a}\right)$ in half-BPS configurations describes selfdual Einstein gravity.
\end{abstract}

PACS numbers: 11.10.Nx, 02.40.Gh, 04.50.th

Keywords: Noncommutative Gauge Theory, Large N Gauge Theory, Emergent Gravity

October 31, 2018

*hsyang@physik.hu-berlin.de 


\section{Introduction}

A noncommutative (NC) spacetime $M$ is obtained by introducing a symplectic structure $B=\frac{1}{2} B_{a b} d y^{a} \wedge$ $d y^{b}$ and then by quantizing the spacetime with its Poisson structure $\theta^{a b} \equiv\left(B^{-1}\right)^{a b}$, treating it as a quantum phase space. That is, for $f, g \in C^{\infty}(M)$,

$$
\{f, g\}=\theta^{a b}\left(\frac{\partial f}{\partial y^{a}} \frac{\partial g}{\partial y^{b}}-\frac{\partial f}{\partial y^{b}} \frac{\partial g}{\partial y^{a}}\right) \Rightarrow-i[\widehat{f}, \widehat{g}] .
$$

According to the Weyl-Moyal map [1, 2], the NC algebra of operators is equivalent to the deformed algebra of functions defined by the Moyal $\star$-product, i.e.,

$$
\widehat{f} \cdot \widehat{g} \cong(f \star g)(y)=\left.\exp \left(\frac{i}{2} \theta^{a b} \partial_{a}^{y} \partial_{b}^{z}\right) f(y) g(z)\right|_{y=z} .
$$

Through the quantization rules (1.1) and (1.2), one can define $\mathrm{NC} \mathbf{R}^{2 n}$ by the following commutation relation

$$
\left[y^{a}, y^{b}\right]_{\star}=i \theta^{a b}
$$

It is well-known [2, 3] that a NC field theory can be identified basically with a matrix model or a large $N$ field theory. This claim is based on the following fact. Let us consider a NC $\mathbb{R}^{2}$ for simplicity,

$$
[x, y]=i \theta
$$

although the same argument equally holds for a $\mathrm{NC} \mathbf{R}^{2 n}$ as it will be shown later. After scaling the coordinates $x \rightarrow \sqrt{\theta} x, y \rightarrow \sqrt{\theta} y$, the NC plane (1.4) becomes the Heisenberg algebra of harmonic oscillator

$$
\left[a, a^{\dagger}\right]=1
$$

It is a well-known fact from quantum mechanics that the representation space of $\mathrm{NC} \mathbf{R}^{2}$ is given by an infinite-dimensional, separable Hilbert space $\mathcal{H}=\{|n\rangle, n=0,1, \cdots\}$ which is orthonormal, i.e., $\langle n \mid m\rangle=\delta_{n m}$ and complete, i.e., $\sum_{n=0}^{\infty}|n\rangle\langle n|=1$. Therefore a scalar field $\widehat{\phi} \in \mathcal{A}_{\theta}$ on the NC plane (1.4) can be expanded in terms of the complete operator basis

$$
\mathcal{A}_{\theta}=\{|m\rangle\langle n|, n, m=0,1, \cdots\},
$$

that is,

$$
\widehat{\phi}(x, y)=\sum_{n, m} M_{m n}|m\rangle\langle n| .
$$

One can regard $M_{m n}$ in (1.7) as components of an $N \times N$ matrix $M$ in the $N \rightarrow \infty$ limit. More generally one may replace $\mathrm{NC} \mathbb{R}^{2}$ by a Riemann surface $\Sigma_{g}$ of genus $g$ which can be quantized via deformation quantization [4]. For a compact Riemann surface $\Sigma_{g}$ with finite area $A\left(\Sigma_{g}\right)$, the matrix representation can be finite-dimensional, e.g., for a fuzzy sphere [5]. In this case, $A\left(\Sigma_{g}\right) \sim \theta N$ but we simply take the limit $N \rightarrow \infty$. We then arrive at the well-known relation:

$$
\text { Scalar field on } \mathrm{NC} \mathbf{R}^{2}\left(\text { or } \Sigma_{g}\right) \Longleftrightarrow N \times N \text { matrix at } N \rightarrow \infty \text {. }
$$


If $\widehat{\phi}$ is a real scalar field, then $M$ should be a Hermitean matrix. We will see that the above relation (1.8) has far-reaching applications to string theory.

The matrix representation (1.7) clarifies why $\mathrm{NC} U(1)$ gauge theory is a large $N$ gauge theory. An important point is that the NC gauge symmetry acts as a unitary transformation on $\mathcal{H}$ for a field $\widehat{\phi} \in \mathcal{A}_{\theta}$ in the adjoint representation of $U(1)$ gauge group

$$
\widehat{\phi} \rightarrow U \widehat{\phi} U^{\dagger}
$$

This NC gauge symmetry $U_{\text {cpt }}(\mathcal{H})$ is so large that $U_{\text {cpt }}(\mathcal{H}) \supset U(N)(N \rightarrow \infty)$ [6, 7], which is rather obvious in the matrix basis (1.6). Therefore the NC gauge theory is essentially a large $N$ gauge theory. It becomes more precise on a NC torus through the Morita equivalence where NC $U(1)$ gauge theory with rational $\theta=M / N$ is equivalent to an ordinary $U(N)$ gauge theory [8]. For this reason, it is not so surprising that $\mathrm{NC}$ electromagnetism shares essential properties appearing in a large $N$ gauge theory such as $S U(N \rightarrow \infty)$ Yang-Mills theory or matrix models.

It is well-known [9] that $1 / N$ expansion of any large $N$ gauge theory using the double line formalism reveals a picture of a topological expansion in terms of surfaces of different genus, which can be interpreted in terms of closed string variables as the genus expansion of string amplitudes. It has been underlain the idea that large $N$ gauge theories have a dual description in terms of gravitational theories in higher dimensions. For example, BFSS matrix model [10], IKKT matrix model [11] and AdS/CFT duality [12]. From the perspective (1.8), the $1 / N$ expansion corresponds to the NC deformation in terms of $\theta / A\left(\Sigma_{g}\right)$.

All these arguments imply that there exists a solid map between a NC gauge theory and a large $N$ gauge theory. In this work we will find a sound realization of this idea. It turns out that the emergent gravity recently found in [13, 14, 15, 16] can be elegantly understood in this framework. Therefore the correspondence between NC field theory and gravity [3] is certainly akin to the gauge/gravity duality in large $N$ limit [10, 11, 12].

This paper is organized as follows. In Section 2 we map NC $U(1)$ gauge theory on $\mathbf{R}_{C}^{d} \times \mathbf{R}_{N C}^{2 n}$ to $U(N \rightarrow \infty)$ Yang-Mills theory on $\mathbb{R}_{C}^{d}$, where $\mathbb{R}_{C}^{d}$ is a $d$-dimensional commutative spacetime while $\mathbb{R}_{N C}^{2 n}$ is a $2 n$-dimensional NC space. The resulting $U(N)$ Yang-Mills theory on $\mathbf{R}_{C}^{d}$ is equivalent to that obtained by the dimensional reduction of $(d+2 n)$-dimensional $U(N)$ Yang-Mills theory onto $\mathbb{R}_{C}^{d}$. In Section 3, we show that the gauge-Higgs system $\left(A_{\mu}, \Phi^{a}\right)$ in the $U(N \rightarrow \infty)$ Yang-Mills theory on $\mathbb{R}_{C}^{d}$ leads to an emergent geometry in the $(d+2 n)$-dimensional spacetime whose metric was determined by Ward [17] a long time ago. In particular, the 10-dimensional gravity for $d=4$ and $n=3$ corresponds to the emergent geometry arising from the 4-dimensional $\mathcal{N}=4$ vector multiplet in the AdS/CFT duality [12]. We further elucidate the emergent gravity in Section 4 by showing that the gauge-Higgs system $\left(A_{\mu}, \Phi^{a}\right)$ in half-BPS configurations describes self-dual Einstein gravity. A notable point is that the emergent geometry arising from the gauge-Higgs system $\left(A_{\mu}, \Phi^{a}\right)$ is closely related to the bubbling geometry in AdS space found in [18]. Finally, in Section 5, we discuss several interesting issues that naturally arise from our construction. 


\section{A Large $N$ Gauge Theory From NC $U(1)$ Gauge Theory}

We will consider a NC $U(1)$ gauge theory on $\mathbb{R}^{D}=\mathbb{R}_{C}^{d} \times \mathbf{R}_{N C}^{2 n}$, where $D$-dimensional coordinates $X^{M}(M=1, \cdots, D)$ are decomposed into $d$-dimensional commutative ones, denoted as $z^{\mu}(\mu=$ $1, \cdots, d)$ and $2 n$-dimensional NC ones, denoted as $y^{a}(a=1, \cdots, 2 n)$, satisfying the relation (1.3). We assume the metric on $\mathbf{R}^{D}=\mathbf{R}_{C}^{d} \times \mathbf{R}_{N C}^{2 n}$ as the following form 1

$$
\begin{aligned}
d s^{2} & =\mathcal{G}_{M N} d X^{M} d X^{N} \\
& =g_{\mu \nu} d z^{\mu} d z^{\nu}+G_{a b} d y^{a} d y^{b} .
\end{aligned}
$$

The action for $D$-dimensional NC $U(1)$ gauge theory is given by

$$
S=\frac{1}{4 g_{Y M}^{2}} \int d^{D} X \sqrt{\operatorname{det} \mathcal{G}} \mathcal{G}^{M P} \mathcal{G}^{N Q}\left(F_{M N}+\Phi_{M N}\right) \star\left(F_{P Q}+\Phi_{P Q}\right),
$$

where the NC field strength $F_{M N}$ is defined by

$$
F_{M N}=\partial_{M} A_{N}-\partial_{N} A_{M}-i\left[A_{M}, A_{N}\right]_{\star} .
$$

The constant two-form $\Phi$ will be taken either 0 or $-B=-\frac{1}{2} B_{a b} d y^{a} \wedge d y^{b}$ with $\operatorname{rank}(B)=2 n$.

Here we will use the background independent prescription [8, 19] where the open string metric $G_{a b}$, the noncommutativity $\theta^{a b}$ and the open string coupling $G_{s}$ are determined by

$$
\theta^{a b}=\left(\frac{1}{B}\right)^{a b}, \quad G_{a b}=-\kappa^{2}\left(B \frac{1}{g} B\right)_{a b}, \quad G_{s}=g_{s} \sqrt{\operatorname{det}^{\prime}\left(\kappa B g^{-1}\right)},
$$

with $\kappa \equiv 2 \pi \alpha^{\prime}$. The closed string metric $g_{a b}$ in Eq.(2.4) is independent of $g_{\mu \nu}$ in Eq.(2.1) and $\operatorname{det}^{\prime}$ denotes a determinant taken along NC directions only in $\mathbf{R}_{N C}^{2 n}$. In terms of these parameters, the couplings are related by

$$
\begin{aligned}
& \frac{1}{g_{Y M}^{2}}=\frac{\kappa^{\frac{4-D}{2}}}{(2 \pi)^{\frac{D-2}{2}} G_{s}}, \\
& \frac{\sqrt{\operatorname{det}^{\prime} G}}{G_{s}}=\frac{\kappa^{n}}{g_{s}|\operatorname{Pf} \theta|} .
\end{aligned}
$$

An important fact is that translations in $\mathrm{NC}$ directions are basically gauge transformations, i.e., $e^{i k \cdot y} \star f(z, y) \star e^{-i k \cdot y}=f(z, y+\theta \cdot k)$ for any $f(z, y) \in C^{\infty}(M)$. This means that translations along $\mathrm{NC}$ directions act as inner derivations of the NC algebra $\mathcal{A}_{\theta}$ :

$$
\left[y^{a}, f\right]_{\star}=i \theta^{a b} \partial_{b} f .
$$

\footnotetext{
${ }^{1}$ Here we can take the $d$-dimensional spacetime metric $g_{\mu \nu}$ with either Lorentzian or Euclidean signature since the signature is inconsequential in our most discussions. But we implicitly assume the Euclidean signature for some other discussions.
} 
Using this relation, each component of $F_{M N}$ can be written as the following forms

$$
\begin{aligned}
& F_{\mu \nu}=i\left[D_{\mu}, D_{\mu}\right]_{\star}, \\
& F_{\mu a}=\theta_{a b}^{-1}\left[D_{\mu}, x^{b}\right]_{\star}=-F_{a \mu}, \\
& F_{a b}=-i \theta_{a c}^{-1} \theta_{b d}^{-1}\left(\left[x^{c}, x^{d}\right]_{\star}-i \theta^{c d}\right),
\end{aligned}
$$

where the covariant derivative $D_{\mu}$ and the covariant coordinate $x^{a}$ are, respectively, defined by

$$
\begin{gathered}
D_{\mu} \equiv \partial_{\mu}-i A_{\mu}, \\
x^{a} \equiv y^{a}+\theta^{a b} A_{b} .
\end{gathered}
$$

Collecting all these facts, one gets the following expression for the action (2.2) with $\Phi=-B 2$

$$
\begin{aligned}
S=\frac{(2 \pi \kappa)^{\frac{4-d}{2}}}{2 \pi g_{s}} \int d^{d} z \sqrt{\operatorname{det} g_{\mu \nu}} \operatorname{Tr}_{\mathcal{H}} & \left(\frac{1}{4} g^{\mu \lambda} g^{\nu \sigma} F_{\mu \nu} \star F_{\lambda \sigma}+\frac{1}{2} g^{\mu \nu} g_{a b} D_{\mu} \Phi^{a} \star D_{\nu} \Phi^{b}\right. \\
& \left.-\frac{1}{4} g_{a c} g_{b d}\left[\Phi^{a}, \Phi^{b}\right]_{\star} \star\left[\Phi^{c}, \Phi^{d}\right]_{\star}\right),
\end{aligned}
$$

where we defined adjoint scalar fields $\Phi^{a} \equiv x^{a} / \kappa$ of mass dimension and

$$
\operatorname{Tr}_{\mathcal{H}} \equiv \int \frac{d^{2 n} y}{(2 \pi)^{n}|\operatorname{Pf} \theta|} .
$$

Note that the number of the adjoint scalar fields is equal to the rank of $\theta^{a b}$. The resulting action (2.13) is not new but rather well-known in NC field theory, e.g., see [19, 20].

The NC algebra (1.3) is equivalent to the Heisenberg algebra of an $n$-dimensional harmonic oscillator in a frame where $\theta^{a b}$ has a canonical form:

$$
\left[a_{i}, a_{j}^{\dagger}\right]=\delta_{i j}, \quad(i, j=1, \cdots, n)
$$

The NC space (1.3) is therefore represented by the infinite-dimensional Hilbert space $\mathcal{H}=\{|\vec{m}\rangle \equiv$ $\left|m_{1}, \cdots, m_{n}\right\rangle ; m_{i}=0,1, \cdots, N \rightarrow \infty$ for $\left.i=1, \cdots, n\right\}$ whose set of eigenvalues forms an $n$ dimensional positive integer lattice. A set of operators in $\mathcal{H}$

$$
\mathcal{A}_{\theta}=\left\{|\vec{m}\rangle\langle\vec{n}| ; m_{i}, n_{i}=0,1, \cdots, N \rightarrow \infty \text { for } i=1, \cdots, n\right\}
$$

can be identified with the generators of a complete operator basis and so any NC field $\phi(z, y) \in \mathcal{A}_{\theta}$ can be expanded in the basis (2.16) as follows,

$$
\phi(z, y)=\sum_{\vec{m}, \vec{n}} \Omega_{\vec{m}, \vec{n}}(z)|\vec{m}\rangle\langle\vec{n}| .
$$

\footnotetext{
${ }^{2}$ If $\Phi=0$ in Eq.(2.2), the only change in Eq. (2.13) is $\left[\Phi^{a}, \Phi^{b}\right] \rightarrow\left[\Phi^{a}, \Phi^{b}\right]-\frac{i}{\kappa^{2}} \theta^{a b}$.
} 
Now we use the 'Cantor diagonal method' to put the $n$-dimensional positive integer lattice in $\mathcal{H}$ into a one-to-one correspondence with the infinite set of natural numbers (i.e., 1-dimensional positive integer lattice): $|\vec{m}\rangle \leftrightarrow|i\rangle, i=1, \cdots, N \rightarrow \infty$. In this one-dimensional basis, Eq.(2.17) is relabeled as the following form

$$
\phi(z, y)=\sum_{i, j} \Omega_{i j}(z)|i\rangle\langle j| .
$$

Following the motivation discussed in the Introduction, we regard $\Omega_{i j}(z)$ in (2.18) as components of an $N \times N$ matrix $\Omega$ in the $N \rightarrow \infty$ limit, which also depend on $z^{\mu}$, the coordinates of $\mathbb{R}_{C}^{d}$. If the field $\phi(z, y)$ is real which is the case for the gauge-Higgs system $\left(A_{\mu}, \Phi^{a}\right)$ in the action (2.13), the matrix $\Omega$ should be Hermitean, but not necessarily traceless. So the $N \times N$ matrix $\Omega(z)$ can be regarded as a field in $U(N \rightarrow \infty)$ gauge theory on $d$-dimensional commutative space $\mathbf{R}_{C}^{d}$, where $\operatorname{Tr}_{\mathcal{H}}$ in (2.14) is identified with the matrix trace over the basis (2.18). All the dependence on NC coordinates is now encoded into $N \times N$ matrices and the noncommutativity in terms of star product is transferred to the matrix product.

Adopting the matrix representation (2.18), the $D$-dimensional NC $U(1)$ gauge theory (2.2) is mapped to the $U(N \rightarrow \infty)$ Yang-Mills theory on $d$-dimensional commutative space $\mathbb{R}_{C}^{d}$. One can see that the resulting $U(N)$ Yang-Mills theory on $\mathbf{R}_{C}^{d}$ in Eq.(2.13) is equivalent to that obtained by the dimensional reduction of $(d+2 n)$-dimensional $U(N)$ Yang-Mills theory onto $\mathbb{R}_{C}^{d}$. It might be emphasized that the map between the $D$-dimensional NC $U(1)$ gauge theory and the $d$-dimensional $U(N \rightarrow \infty)$ Yang-Mills theory is "exact" and thus the two theories should describe a completely equivalent physics. For example, we can recover the $D$-dimensional NC $U(1)$ gauge theory on $\mathbf{R}_{C}^{d} \times$ $\mathbb{R}_{N C}^{2 n}$ from the $d$-dimensional $U(N \rightarrow \infty)$ Yang-Mills theory on $\mathbb{R}_{C}^{d}$ by recalling that the number of adjoint Higgs fields in the $U(N)$ Yang-Mills theory is equal to the dimension of the extra NC space $\mathbb{R}_{N C}^{2 n}$ and by applying the dictionary in Eqs.(2.8)-(2.10).

One can introduce linear algebraic conditions of $D$-dimensional field strengths $F_{M N}$ as a higher dimensional analogue of 4-dimensional self-duality equations such that the Yang-Mills equations in the action (2.2) follow automatically. These are of the following type [21, 22]

$$
\frac{1}{2} T_{M N P Q} F_{P Q}=\lambda F_{M N}
$$

with a constant 4-form tensor $T_{M N P Q}$. The relation (2.19) clearly implies via the Bianchi identity $D_{[M} F_{P Q]}=0$ that the Yang-Mills equations are satisfied provided $\lambda$ is nonzero. For $D>4$, the 4form tensor $T_{M N P Q}$ cannot be invariant under $S O(D)$ transformations and the equation (2.19) breaks the rotational symmetry to a subgroup $H \subset S O(D)$. Thus the resulting first order equations can be classified by the unbroken symmetry $H$ under which $T_{M N P Q}$ remain invariant [21, 22]. It was also shown [23] that the first order linear equations above are closely related to supersymmetric states, i.e., BPS states in higher dimensional Yang-Mills theories.

The equivalence between $D$ - and $d$-dimensional gauge theories can be effectively used to classify classical solutions in the $d$-dimensional $U(N)$ Yang-Mills theory (2.13). The group theoretical 
classification [21], integrability condition [22] and BPS states [23] for the $D$-dimensional first-order equations (2.19) can be directly translated into the properties of the gauge-Higgs system $\left(A_{\mu}, \Phi^{a}\right)$ in the $d$-dimensional $U(N)$ gauge theory (2.13). These classifications will also be useful to classify the geometries emerging from the gauge-Higgs system $\left(A_{\mu}, \Phi^{a}\right)$ in the $U(N \rightarrow \infty)$ Yang-Mills theory (2.13), which will be discussed in the next section. Unfortunately, the $D=10$ case is missing in $[21,22,23]$ which is the most interesting case $(d=4$ and $n=3)$ related to the AdS/CFT duality.

\section{Emergent Geometry From NC Gauge Theory}

Let us first recapitulate the result in [17]. It turns out that the Ward's construction perfectly fits with the emergent geometry arising from the gauge-Higgs system $\left(A_{\mu}, \Phi^{a}\right)$ in the $U(N \rightarrow \infty)$ Yang-Mills theory (2.13). Suppose that we have gauge fields on $\mathbf{R}_{C}^{d}$ taking values in the Lie algebra of volumepreserving vector fields on an $m$-dimensional manifold $M$ [24, 25]. In other words, the gauge group $G=S \operatorname{Dif} f(M)$. The gauge covariant derivative is given by Eq.(2.11), but the $A_{\mu}(z)$ are now vector fields on $M$, also depending on $z^{\mu} \in \mathbb{R}_{C}^{d}$. The other ingredient in [17] consists of $m$ Higgs fields $\Phi^{a}(z) \in \operatorname{sdiff}(M)$, the Lie algebra of $\operatorname{SDiff}(M)$, for $a=1, \cdots, m$. The idea [24, 25] is to specify that

$$
f^{-1}\left(D_{1}, \cdots, D_{d}, \Phi_{1}, \cdots, \Phi_{m}\right)
$$

forms an orthonormal frame and hence defines a metric on $\mathbf{R}_{C}^{d} \times M$ with a volume form $\nu=d^{d} z \wedge \omega$. Here $f$ is a scalar, a conformal factor, defined by

$$
f^{2}=\omega\left(\Phi_{1}, \cdots, \Phi_{m}\right)
$$

The result in [24, 25] immediately implies that the gauge-Higgs system $\left(A_{\mu}, \Phi^{a}\right)$ leads to a metric on the $(d+m)$-dimensional space $\mathbb{R}_{C}^{d} \times M$. A local coordinate expression for this metric is easily obtained from Eq.(3.1). Let $y^{a}$ be local coordinates on $M$. So $A_{\mu}(z)$ and $\Phi_{a}(z)$ have the form

$$
A_{\mu}(z)=A_{\mu}^{a}(z, y) \frac{\partial}{\partial y^{a}}, \quad \Phi_{a}(z)=\Phi_{a}^{b}(z, y) \frac{\partial}{\partial y^{b}},
$$

where the $y$-dependence, originally hidden in the Lie algebra of $G=\operatorname{SDiff}(M)$, now explicitly appears in the coefficients $A_{\mu}^{a}$ and $\Phi_{a}^{b}$. Let $V_{b}^{a}$ denote the inverse of the $m \times m$ matrix $\Phi_{a}^{b}$, and let $\mathbf{A}^{a}$ denote the 1-form $A_{\mu}^{a} d z^{\mu}$. Then the metric is [17]

$$
d s^{2}=f^{2} \delta_{\mu \nu} d z^{\mu} d z^{\nu}+f^{2} \delta_{a b} V_{c}^{a} V_{d}^{b}\left(d y^{c}-\mathbf{A}^{c}\right)\left(d y^{d}-\mathbf{A}^{d}\right) .
$$

It will be shown later that the choice of the volume form $\omega$ for the conformal factor (3.2) corresponds to that of a particular conformally flat background although we mostly assume a flat volume form, i.e., $\omega \sim d y^{1} \wedge \cdots \wedge d y^{2 n}$, unless explicitly specified.

The gauge and Higgs fields in Eq. (3.3) are not arbitrary but must be subject to the Yang-Mills equations, for example, derived from the action (2.13), which are, in most cases, not completely 
integrable. Hence to completely determine the geometric structure emerging from the gauge-Higgs system $\left(A_{\mu}, \Phi^{a}\right)$ is as much difficult as solving the Einstein equations in general. But the self-dual Yang-Mills equations in four dimensions or Eq.(2.19) in general are, in some sense, "completely solvable". Thus the metric (3.4) for these cases might be completely determined. Let us discuss two notable examples. See [17] for more examples describing 4-dimensional self-dual Einstein gravity.

- Case $d=0, m=4$ : This case was dealt with in detail in [25, 26, 27]. It was proved that the self-dual Einstein equations are equivalent to the self-duality equations

$$
\left[\Phi_{a}, \Phi_{b}\right]= \pm \frac{1}{2} \varepsilon_{a b c d}\left[\Phi_{c}, \Phi_{d}\right]
$$

on the four Higgs fields $\Phi_{a}$. Furthermore reinterpreting $n$ of the $\Phi_{a}$ 's as $D_{\mu}$ leads to the case $d=$ $n, m=4-n$. In Section 5, we will discuss the physical meaning about the interpretation $\Phi_{a} \mapsto D_{\mu}$.

- Case $d=3, m=1$ : Here $M$ is one-dimensional, so the Lie algebra of vector fields on $M$ is the Virasoro algebra. Thus $A_{\mu}$ and $\Phi$ are now real-valued vector fields on $M$ which must be independent of $y$ to preserve the volume form $\nu=d^{3} z \wedge d y$ [27]. The metric (3.4) reduces to

$$
d s^{2}=\Phi d \vec{z} \cdot d \vec{z}+\Phi^{-1}\left(d y-A_{\mu} d z^{\mu}\right)^{2}
$$

and has a Killing vector $\partial / \partial y$. In this case, the self-duality equations (3.5) reduce to the Abelian Bogomol'nyi equations, $\nabla \times \vec{A}=\nabla \Phi$, and the metric (3.6) describes a gravitational instanton [28].

Recently we showed in [15, 16] for the $d=0$ and $m=4$ case that self-dual electromagnetism in $\mathrm{NC}$ spacetime is equivalent to self-dual Einstein gravity and the metric is precisely given by Eq.(3.4). A key observation [16] was that the self-dual system (3.5) defined by vector fields on $M$ can be derived from the action (2.2) or (2.13) for slowly varying fields, where all $\star$-commutators between NC fields are approximated by the Poisson bracket (1.1). An important point in NC geometry is that the adjoint action of (covariant) coordinates with respect to star product can be identified with (generalized) vector fields on some (curved) manifold [15, 16], as the trivial case was already used in Eq.(2.7). In the end, a D-dimensional manifold described by the metric (3.4) corresponds to an emergent geometry arising from the gauge-Higgs system in Eq.(3.3). Now we will show in a general context how the nontrivial geometry (3.4) emerges from the gauge-Higgs system $\left(A_{\mu}, \Phi^{a}\right)$ in the action (2.13).

Let us collectively denote the covariant derivatives $D_{\mu}$ in (2.11) and the Higgs fields $D_{a} \equiv$ $-i \kappa B_{a b} \Phi^{b}=-i\left(B_{a b} y^{b}+A_{a}\right)$ in (2.12) as $D_{A}(z, y)$. Therefore $D_{A}(z, y)$ transform covariantly under $\mathrm{NC} U(1)$ gauge transformations

$$
D_{A}(z, y) \rightarrow g(z, y) \star D_{A}(z, y) \star g^{-1}(z, y)
$$

Define the adjoint action of $D_{A}(z, y)$ with respect to star product acting on any NC field $f(z, y) \in \mathcal{A}_{\theta}$ :

$$
\operatorname{ad}_{D_{A}}[f] \equiv\left[D_{A}, f\right]_{\star}
$$


Then it is easy to see [16] that the above adjoint action satisfies the Leibniz rule and the Jocobi identity, i.e.,

$$
\begin{aligned}
& {\left[D_{A}, f \star g\right]_{\star}=\left[D_{A}, f\right]_{\star} \star g+f \star\left[D_{A}, g\right]_{\star},} \\
& {\left[D_{A},\left[D_{B}, f\right]_{\star}\right]_{\star}-\left[D_{B},\left[D_{A}, f\right]_{\star}\right]_{\star}=\left[\left[D_{A}, D_{B}\right]_{\star}, f\right]_{\star} .}
\end{aligned}
$$

These properties imply that $\operatorname{ad}_{D_{A}}$ can be identified with 'generalized' vector fields or Lie derivatives acting on the algebra $\mathcal{A}_{\theta}$, which can be viewed as a gauge covariant generalization of the inner derivation (2.7). Note that the generalized vector field in Eq.(3.8) is a kind of general higher order differential operators in [29]. Indeed it turns out that they constitute a generalization of volume preserving diffeomorphisms to $\star$-differential operators acting on $\mathcal{A}_{\theta}$ (see Eqs.(4.1) and (4.2) in [7]).

In particular, the generalized vector fields in Eq.(3.8) reduce to usual vector fields in the commutative, i.e. $\mathcal{O}(\theta)$, limit:

$$
\begin{aligned}
\operatorname{ad}_{D_{A}}[f] & =i \theta^{a b} \frac{\partial D_{A}}{\partial y^{a}} \frac{\partial f}{\partial y^{b}}+\cdots=i\left\{D_{A}, f\right\}+\mathcal{O}\left(\theta^{3}\right) \\
& \equiv V_{A}^{a}(z, y) \partial_{a} f(z, y)+\mathcal{O}\left(\theta^{3}\right)
\end{aligned}
$$

where we defined $\left[\partial_{\mu}, f\right]_{\star}=\partial_{\mu} f$. Note that the vector fields $V_{A}(z, y)=V_{A}^{a}(z, y) \partial_{a}$ are exactly of the same form as Eq. (3.3) and belong to the Lie algebra of volume preserving diffeomorphisms, as precisely required in the Ward construction (3.1), since they are all divergence free, i.e., $\partial_{a} V_{A}^{a}=0$. Thus the vector fields $f^{-1} V_{A}(z, y)$ for $A=1, \cdots, D$ can be identified with the orthonormal frame (3.1) defining the metric (3.4). It should be emphasized that the emergent gravity (3.4) arises from a general, not necessarily self-dual, gauge-Higgs system $\left(A_{\mu}, \Phi^{a}\right)$ in the action (2.13).

Note that

$$
\left[D_{A}, D_{B}\right]_{\star}=-i\left(F_{A B}-B_{A B}\right)
$$

where the NC field strength $F_{A B}$ is given by Eq.(2.3). Then the Jacobi identity (3.10) leads to the following identity for a constant $B_{A B}$

$$
\operatorname{ad}_{\left[D_{A}, D_{B}\right]_{\star}}=-i \operatorname{ad}_{F_{A B}}=\left[\operatorname{ad}_{D_{A}}, \operatorname{ad}_{D_{B}}\right]_{\star} .
$$

The inner derivation (3.11) in commutative limit is reduced to the well-known map $C^{\infty}(M) \rightarrow$ $T M: f \mapsto X_{f}$ between the Poisson algebra $\left(C^{\infty}(M),\{\cdot, \cdot\}\right)$ and vector fields in $T M$ defined by $X_{f}(g)=\{g, f\}$ for any smooth function $g \in C^{\infty}(M)$. The Jacobi identity for the Poisson algebra $\left(C^{\infty}(M),\{\cdot, \cdot\}\right)$ then leads to the Lie algebra homomorphism

$$
X_{\{f, g\}}=-\left[X_{f}, X_{g}\right]
$$

where the right-hand side is defined by the Lie bracket between Hamiltonian vector fields. One can check by identifying $f=D_{A}$ and $g=D_{B}$ that the Lie algebra homomorphism (3.14) correspond 
to the commutative limit of the Jacobi identity (3.10). That is, one can deduce from Eq. (3.14) the following identity

$$
X_{F_{A B}}=-\left[V_{A}, V_{B}\right]
$$

using the relation $\left\{D_{A}, D_{B}\right\}=-F_{A B}+B_{A B}$ and $X_{D_{A}}=i V_{A}$.

Using the homomorphism (3.15), one can translate the generalized self-duality equation (2.19) into the structure equation between vector fields

$$
\frac{1}{2} T_{A B C D} F_{C D}=\lambda F_{A B} \quad \Leftrightarrow \quad \frac{1}{2} T_{A B C D}\left[V_{C}, V_{D}\right]=\lambda\left[V_{A}, V_{B}\right]
$$

Therefore a D-dimensional NC gauge field configuration satisfying the first-order system defined by the left-hand side of Eq.(3.16) is isomorphic to a D-dimensional emergent geometry defined by the right-hand side of Eq.(3.16) whose metric is given by Eq.(3.4). For example, in four dimensions where $T_{A B C D}=\varepsilon_{A B C D}$ and $\lambda= \pm 1$, the right-hand side of Eq.(3.16) is precisely equal to Eq.(3.5) describing gravitational instantons $[24,25,26,27]$. This proves, as first shown in [15, 16], that selfdual NC electromagnetism is equivalent to self-dual Einstein gravity. Note that the Einstein gravity described by the metric (3.4) arises from the commutative, i.e., $\mathcal{O}(\theta)$ limit. Therefore it is natural to expect that the higher order differential operators in Eq.(3.11), e.g. $\mathcal{O}\left(\theta^{3}\right)$, give rise to higher order gravity [16]. We will further discuss the derivative correction in Section 5.

The 10-dimensional metric (3.4) for $d=4$ and $n=3(m=6)$ is particularly interesting since it corresponds to an emergent geometry arising from the 4-dimensional $\mathcal{N}=4$ vector multiplet in the AdS/CFT duality. Note that the gravity in the AdS/CFT duality is an emergent phenomenon arising from particle interactions in a gravityless, lower-dimensional spacetime. As a famous example, the type IIB supergravity (or more generally the type IIB superstring theory) on $A d S_{5} \times \mathbf{S}^{5}$ is emergent from the 4-dimensional $\mathcal{N}=4$ supersymmetric $U(N)$ Yang-Mills theory [12] 3 In our construction, $N \times N$ matrices are mapped to vector fields on some manifold $M$, so the vector fields in Eq. (3.3) correspond to master fields of large $N$ matrices [30], in other words, $\left(A_{\mu}, \Phi^{a}\right) \sim N^{2}$. According to the AdS/CFT duality, we thus expect that the metric (3.4) describes a deformed geometry induced by excitations of the gauge and Higgs fields in the action (2.13). For example, we may look for $1 / 2$ BPS geometries in type IIB supergravity that arise from chiral primaries of $\mathcal{N}=4$ super YangMills theory [18]. Recently this kind of BPS geometries, the so-called bubbling geometry in AdS space, with a particular isometry was completely determined in [18], where the $A d S_{5} \times \mathbf{S}^{5}$ geometry emerges from the simplest and most symmetric configuration. In next section we will illustrate such kind of bubbling geometry described by the metric (3.4) by considering self-dual configurations in the gauge-Higgs system.

\footnotetext{
${ }^{3}$ The overall $U(1)=U(N) / S U(N)$ factor actually corresponds to the overall position of D3-branes and may be ignored when considering dynamics on the branes, thereby leaving only an $S U(N)$ gauge symmetry.
} 


\section{Self-dual Einstein Gravity From Large $N$ Gauge Theory}

In the previous section we showed that the Ward's metric (3.4) naturally emerges from the $D$-dimensional NC $U(1)$ gauge fields $A_{M}$ on $\mathbb{R}_{C}^{d} \times \mathbf{R}_{N C}^{2 n}$ or equivalently the gauge-Higgs system $\left(A_{\mu}, \Phi^{a}\right)$ in $d$ dimensional $U(N)$ gauge theory on $\mathbf{R}_{C}^{d}$. So, if an explicit solution for $A_{M}$ or $\left(A_{\mu}, \Phi^{a}\right)$ is known, the corresponding metric (3.4) is, in principle, exactly determined. However, it is extremely difficult to get a general solution by solving the equations of motion for the action (2.2) or (2.13). Instead we may try to solve a more simpler system such as the first-order equations (2.19), which are morally believed to be 'exactly solvable' in most cases. In this section we will further elucidate the emergent gravity arising from gauge fields by showing that the gauge-Higgs system $\left(A_{\mu}, \Phi^{a}\right)$ in half-BPS configurations describes self-dual Einstein gravity. Since the case for $D=4$ and $n=2$ has been extensively discussed in [14, 15, 16], we will consider the other cases for $D \geq 4$. For simplicity, the metrics in the action (2.13) are supposed to be the form already used in Eq.(3.4); $g_{\mu \nu}=\delta_{\mu \nu}$ and $g_{a b}=\delta_{a b}$.

Note that the action (2.2) or (2.13) contains a background $B$, due to a uniform condensation of gauge fields in a vacuum. But we will require a rapid fall-off of fluctuating fields around the background at infinity in $\mathbb{R}^{D}$ as usual 4 Our boundary condition is $F_{M N} \rightarrow 0$ at infinity. Eq.(2.10) then requires that $\left[x^{a}, x^{b}\right]_{\star} \rightarrow i \theta^{a b}$ at $|y| \rightarrow \infty$. Thus the coordinates $y^{a}$ in (2.12) are vacuum expectation values of $x^{a}$ characterizing the uniform condensation of gauge fields [16]. This condensation of the $B$-fields endows the $\star$-algebra $\mathcal{A}_{\theta}$ with a remarkable property that translations act as an inner automorphism of the algebra $\mathcal{A}_{\theta}$ as shown in Eq.(2.7). But the gauge symmetry on NC spacetime requires the covariant coordinates $x^{a}$ in Eq.(2.12) instead of $y^{a}$ [31]. The inner derivation $\operatorname{ad}_{D_{a}}$ in Eq. (3.8) is then a 'dual element' related to the coordinate $x^{a}$. This is also true for the covariant derivatives $D_{\mu}$ in (2.11) since they are related to $D_{a}=-i B_{a b} x^{b}$ by the 'matrix $T$-duality'; $D_{a} \mapsto D_{\mu}$, as will be explained in Section 5.

It is very instructive to take an analogy with quantum mechanics. Quantum mechanical time evolution in Heisenberg picture is defined as an inner automorphism of the Weyl algebra obtained from a quantum phase space

$$
f(t)=e^{i H t} f(0) e^{-i H t}
$$

and its evolution equation is of the form (3.8)

$$
\frac{d f(t)}{d t}=i[H, f(t)]
$$

Here we liberally interpret $D_{A}(z, y)$ in Eq.(3.8) as 'multi-Hamiltonians' determining the spacetime evolution in $\mathbf{R}^{D}$. Then it is quite natural to interpret Eq.(3.8) as a spacetime evolution equation determined by the "covariant Hamiltonians" $D_{A}(z, y)$.

\footnotetext{
${ }^{4}$ In the matrix representation (2.18), this means that matrix components $\Omega_{i j}(z)$ for the fluctuations are rapidly vanishing for $i, j=N \rightarrow \infty$ as well as for $|z| \rightarrow \infty$, since roughly $N \sim \vec{y} \cdot \vec{y}$.
} 
Let us be more precise about the meaning of the spacetime evolution. If the Hamiltonian is slightly deformed, $H \rightarrow H+\delta H$, the time evolution of a system is correspondingly changed. Likewise, the fluctuation of gauge fields $A_{M}$ or $\left(A_{\mu}, \Phi^{a}\right)$ around the background specified by $y^{a}$ 's changes $D_{A}(z, y)$, which in turn induces a deformation of the background spacetime according to Eq.(3.11). This is precisely the picture about the emergent geometry in [15, 16] and also a dependable interpretation of the Ward's geometry (3.4). A consistent picture related to the AdS/CFT duality was also observed in the last of Section 3.

For the above reason, all equations in the following will be understood as inner derivations acting on $\mathcal{A}_{\theta}$ like as (3.8). The adjoint action defined in this way naturally removes a contribution from the background in the action (2.2) or (2.13) [15]. For example, the first equation in (4.1) can be consistent only in this way since the left hand side goes to zero at infinity but the right hand side becomes $\sim \theta / \kappa^{2}$. It might be remarked that this is the way to define the equations of motion in the background independent formulation [8, 19] and thus it should be equivalent to the usual NC prescription with $\Phi_{M N}=0$.

\section{1 $D=4$ and $n=1$}

$\mathrm{NC}$ instanton solutions in this case were constructed in [32]. As was proved in Eq.(3.16), NC $U(1)$ instantons are in general equivalent to gravitational instantons. We thus expect that the NC selfduality equations for $D=4$ and $n=1$ are mapped to self-dual Einstein equations. We will show that the gauge-Higgs system $\left(A_{\mu}, \Phi^{a}\right)$ in this case is mapped to two-dimensional $U(\infty)$ chiral model, whose equations of motion are equivalent to the Plebański form of the self-dual Einstein equations [33, 17, 34].

We showed in Section 2 that 4-dimensional NC $U(1)$ gauge theory on $\mathbb{R}_{C}^{2} \times \mathbb{R}_{N C}^{2}$ is mapped to 2-dimensional $U(N \rightarrow \infty)$ gauge theory with the action (2.13). The 4-dimensional self-duality equations now become the $U(N \rightarrow \infty)$ Hitchin equations on $\mathbf{R}_{C}^{2}$ :

$$
F_{\mu \nu}= \pm \frac{1}{2} \varepsilon_{\mu \nu}\left[\Phi, \Phi^{\dagger}\right], \quad D_{\mu} \Phi= \pm i \varepsilon_{\mu \nu} D_{\nu} \Phi
$$

where $\Phi=\Phi_{1}+i \Phi_{2}$. Note that the above equations also arise as zero-energy solutions in $U(N)$ Chern-Simons gauge theory coupled to a nonrelativistic complex scalar field in the adjoint representation [35]. It was shown in [36] that the self-dual system in Eq. (4.1) is completely solvable in terms of Uhlenbeck's uniton method. A NC generalization of Eq.4.1), the Hitchin's equations on $\mathbb{R}_{N C}^{2}$, was also considered in [37] with very parallel results to the commutative case. We will briefly discuss the NC Hitchin's equations in Section 5.

The equations (4.1) for the self-dual case (with + sign) can be elegantly combined into a zerocurvature condition [35, 36] for the new connections defined by

$$
\mathcal{A}_{+}=A_{+}+\Phi, \quad \mathcal{A}_{-}=A_{-}-\Phi^{\dagger}
$$

\footnotetext{
${ }^{5}$ Here we will relax the reality condition of the fields $\left(A_{\mu}, \Phi^{a}\right)$ and complexify them.
} 
with $A_{ \pm}=A_{1} \pm i A_{2}$ :

$$
\mathcal{F}_{+-}=\partial_{+} \mathcal{A}_{-}-\partial_{-} \mathcal{A}_{+}-i\left[\mathcal{A}_{+}, \mathcal{A}_{-}\right]=0
$$

where $\partial_{ \pm}=\partial_{1} \pm i \partial_{2}$. Thus the new gauge fields should be a pure gauge, that is, $\mathcal{A}_{ \pm}=i g^{-1} \partial_{ \pm} g$ for some $g \in G L(N, \mathbb{C})$. Thus we can choose them to be zero, viz.

$$
A_{+}=-\Phi, \quad A_{-}=\Phi^{\dagger}
$$

Then the self-dual equations (4.1) reduce to

$$
\begin{aligned}
& \partial_{+} \Phi^{\dagger}+\partial_{-} \Phi+2 i\left[\Phi, \Phi^{\dagger}\right]=0, \\
& \partial_{+} \Phi^{\dagger}-\partial_{-} \Phi=0 .
\end{aligned}
$$

Introducing another gauge fields $C_{+}=-2 \Phi$ and $C_{-}=2 \Phi^{\dagger}$, Eq.(4.5) also becomes the zero-curvature condition, hence $C_{ \pm}$are a pure gauge or

$$
\Phi=-\frac{i}{2} h^{-1} \partial_{+} h, \quad \Phi^{\dagger}=\frac{i}{2} h^{-1} \partial_{-} h .
$$

A group element $h(z)$ defines a map from $\mathbb{R}_{C}^{2}$ to $G L(N, \mathbb{C})$ group, which is contractible to the map from $\mathbf{R}_{C}^{2}$ to $U(N) \subset G L(N, \mathbb{C})$. Then Eq.(4.6) implies that $h(z)$ satisfies the equation in the twodimensional $U(N)$ chiral model [35, 36]

$$
\partial_{+}\left(h^{-1} \partial_{-} h\right)+\partial_{-}\left(h^{-1} \partial_{+} h\right)=0 .
$$

Eq. (4.8) is the equation of motion derived from the two-dimensional $U(N)$ chiral model governed by the following Euclidean action

$$
S=\frac{1}{2} \int d^{2} z \operatorname{Tr} \partial_{\mu} h^{-1} \partial_{\nu} h \delta^{\mu \nu} .
$$

A remarkable (mysterious) fact has been known [33, 17, 34] that in the $N \rightarrow \infty$ limit the chiral model (4.9) describes a self-dual spacetime whose equation of motion takes the Plebański form of self-dual Einstein equations [38]. Thus, including the case of $D=4$ and $n=2$ in [14, 15, 16], we have confirmed Eq.(3.16) stating that the 4-dimensional self-dual system in the action (2.2) or (2.13) in general describes the self-dual Einstein gravity where self-dual metrics are given by Eq.(3.4).

\section{2 $D=6$ and $n=1$}

Our current work has been particularly motivated by this case since it was already shown in [39] that $S U(N)$ Yang-Mills instantons in the $N \rightarrow \infty$ limit are gravitational instantons too. Since NC $U(1)$ instantons are also gravitational instantons as we showed before, it implies that there should be a close relationship between $S U(N)$ Yang-Mills instantons and $N C U(1)$ instantons. A basic observation was the relation (1.8), which leads to the sound realization in Eq.(2.13). But we will simply follow the 
argument in [39] for the gauge group $G=U(N)$; in the meantime, we will confirm the results for the emergent geometry from NC gauge fields.

Let us look at the instanton solution in $U(N)$ Yang-Mills theory. The self-duality equation is given by

$$
F_{\mu \nu}= \pm \frac{1}{2} \varepsilon_{\mu \nu \alpha \beta} F_{\alpha \beta}
$$

where the field strength is defined by

$$
F_{\mu \nu}=\partial_{\mu} A_{\nu}-\partial_{\nu} A_{\mu}-i\left[A_{\mu}, A_{\nu}\right]
$$

In terms of the complex coordinates and the complex gauge fields defined by

$$
\begin{array}{ll}
z_{1}=\frac{1}{2}\left(x^{2}+i x^{1}\right), & z_{2}=\frac{1}{2}\left(x^{4}+i x^{3}\right), \\
A_{z_{1}}=A_{2}-i A_{1}, & A_{z_{2}}=A_{4}-i A_{3},
\end{array}
$$

Eq.(4.10) can be written as

$$
\begin{aligned}
& F_{z_{1} z_{2}}=0=F_{\bar{z}_{1} \bar{z}_{2}}, \\
& F_{z_{1} \bar{z}_{1}} \mp F_{z_{2} \bar{z}_{2}}=0 .
\end{aligned}
$$

Now let us consider the anti-self-dual (ASD) case. We first notice that $F_{z_{1} z_{2}}=0$ implies that there exists a $u(N)$-valued function $g$ such that $A_{z_{a}}=i g^{-1} \partial_{z_{a}} g(a=1,2)$. Therefore one can choose a gauge

$$
A_{z_{a}}=0 .
$$

Under the gauge (4.14), the ASD equations lead to

$$
\begin{aligned}
& \partial_{\bar{z}_{1}} A_{\bar{z}_{2}}-\partial_{\bar{z}_{2}} A_{\bar{z}_{1}}-i\left[A_{\bar{z}_{1}}, A_{\bar{z}_{2}}\right]=0, \\
& \partial_{z_{1}} A_{\bar{z}_{1}}+\partial_{z_{2}} A_{\bar{z}_{2}}=0 .
\end{aligned}
$$

First notice a close similarity with Eqs.(4.5) and (4.6). Eq.(4.16) can be solved by introducing a $u(N)$-valued function $\Phi$ such that

$$
A_{\bar{z}_{1}}=-\partial_{z_{2}} \Phi, \quad A_{\bar{z}_{2}}=\partial_{z_{1}} \Phi
$$

Substituting (4.17) into (4.15) one finally gets

$$
\left(\partial_{z_{1}} \partial_{\bar{z}_{1}}+\partial_{z_{2}} \partial_{\bar{z}_{2}}\right) \Phi-i\left[\partial_{z_{1}} \Phi, \partial_{z_{2}} \Phi\right]=0 .
$$

Adopting the correspondence (1.8), we now regard $\Phi \in u(N) \otimes C^{\infty}\left(\mathbf{R}^{4}\right)$ in Eq.(4.18) as a smooth function on $\mathbf{R}^{4} \times \Sigma_{g}$, i.e., $\Phi=\Phi\left(x^{\mu}, p, q\right)$ where $(p, q)$ are local coordinates of a two-dimensional Riemann surface $\Sigma_{g}$. Moreover, a Lie algebra commutator is replaced by the Poisson bracket (1.1)

$$
\{f, g\}=\frac{\partial f}{\partial q} \frac{\partial g}{\partial p}-\frac{\partial f}{\partial p} \frac{\partial g}{\partial q},
$$


that is,

$$
\left[\Phi_{1}, \Phi_{2}\right] \rightarrow i\left\{\Phi_{1}, \Phi_{2}\right\}
$$

where we absorbed $\theta$ into the coordinates $(p, q)$. After all, the ASD Yang-Mills equation (4.18) in the large $N$ limit is equivalent to a single nonlinear equation in six dimensions parameterized by $\left(x^{\mu}, p, q\right)$ :

$$
\left(\partial_{z_{1}} \partial_{\bar{z}_{1}}+\partial_{z_{2}} \partial_{\bar{z}_{2}}\right) \Phi+\left\{\partial_{z_{1}} \Phi, \partial_{z_{2}} \Phi\right\}=0
$$

Since Eq.(4.20) is similar to the well-known second heavenly equation [38], it was called in [39] as the six dimensional version of the second heavenly equation.

Starting from $U(N)$ Yang-Mills instantons in four dimensions, we arrived at the nonlinear differential equation for a single function in six dimensions. It is important to notice that the resulting six-dimensional theory is a NC field theory since the Riemann surface $\Sigma_{g}$ carries a symplectic structure inherited from the $u(N)$ Lie algebra through Eq.4.19) and it can be quantized in general via deformation quantization [4]. Since the function $\Phi$ in (4.20) is a master field of $N \times N$ matrices [30], so $\Phi \sim N^{2}$, the AdS/CFT duality [12] implies that the master field $\Phi$ describes a six-dimensional emergent geometry induced by Yang-Mills instantons.

To see the emergent geometry, consider an appropriate symmetry reduction of Eq.(4.20) to show that it describes self-dual gravity in four dimensions. There are many reductions from six to four dimensional subspace leading to self-dual four-manifolds [39]. A common feature is that the four dimensional subspace necessarily contains the NC Riemann surface $\Sigma_{g}$. We will show later how the symmetry reduction naturally arises from the BPS condition in six dimensions. As a specific example, we assume the following symmetry,

$$
\partial_{z_{1}} \Phi=\partial_{\bar{z}_{1}} \Phi, \quad \partial_{z_{2}} \Phi=\partial_{\bar{z}_{2}} \Phi
$$

or

$$
\Phi\left(z_{1}, z_{2}, \bar{z}_{1}, \bar{z}_{2}, p, q\right)=\Lambda\left(z_{1}+\bar{z}_{1} \equiv x, z_{2}+\bar{z}_{2} \equiv y, p, q\right) .
$$

Then Eq.(4.20) is precisely equal to the Husain's equation [34] which is the reduction of self-dual Einstein equations to the $\operatorname{sdiff}\left(\Sigma_{g}\right)$ chiral field equations in two dimensions:

$$
\Lambda_{x x}+\Lambda_{y y}+\Lambda_{x q} \Lambda_{y p}-\Lambda_{x p} \Lambda_{y q}=0
$$

Note that we already encountered in Section 4.1 the two-dimensional sdif $f\left(\Sigma_{g}\right)$ chiral field equations since $\operatorname{sdiff}\left(\Sigma_{g}\right) \cong u(N)$ according to the correspondence (1.8). We showed in [16] that Eq.(4.23) can be transformed to the first heavenly equation [38] which is a governing equation of self-dual Einstein gravity. In the end we conclude that self-dual $U(N)$ Yang-Mills theory in the large $N$ limit is equivalent to self-dual Einstein gravity.

Now it is easy to see that the self-dual Einstein equation (4.23) is coming from a 1/2 BPS equation in six dimensions (see Eq.(34) in [23]) defined by the first-order equation (2.19). According to our construction, the six-dimensional NC $U(1)$ gauge theory (2.2) is equivalent to the four-dimensional 
$U(N)$ gauge theory (2.13). Therefore six-dimensional BPS equations can be equivalently described by the gauge-Higgs system $\left(A_{\mu}, \Phi^{a}\right)$ in the action (2.13). Let us newly denote the NC coordinates $y^{1}, y^{2}$ and commutative ones $z^{3}, z^{4}$ as $u^{\alpha}, \alpha=1,2,3,4$ while $z^{1}, z^{2}$ as $v^{A}, A=1,2$. The $1 / 2$ BPS equations, Eq.(34), in [23] can then be written as the following form

$$
\begin{aligned}
& F_{\alpha \beta}= \pm \frac{1}{2} \varepsilon_{\alpha \beta \gamma \delta} F_{\gamma \delta}, \\
& F_{\alpha A}=F_{A B}=0 .
\end{aligned}
$$

Using Eqs.(2.8)-(2.10), the above equations can be rewritten in terms of $\left(A_{\mu}, \Phi^{a}\right)$ where the constant term in (2.10) can simply be dropped for the reason explained before.

$F_{A B}=0$ in Eq.4.25) can be solved by $A_{B}=0(B=1,2)$ and then $F_{\alpha A}=0$ demand that the gauge fields $A_{\alpha}$ should not depend on $v^{A}$. Thereby Eq.(4.24) precisely reduces to the self-duality equation (4.1) for $D=4$ and $n=1$. The symmetry reduction considered above is now understood as the condition (4.25); in specific, the coordinates $v^{A}$ correspond to $i\left(z_{1}-\bar{z}_{1}\right)$ and $i\left(z_{2}-\bar{z}_{2}\right)$ for the reduction (4.22). However there are many different choices taking a four-dimensional subspace in Eq.(4.24) which are related by $S O(6)$ rotations [23]. Unless $v^{A} \in\left(y^{1}, y^{2}\right)$, that is, Eq. (44.24) becomes commutative Abelian equations in which there is no non-singular solution, Eqs.(4.24) and (4.25) reduce to four-dimensional self-dual Einstein equations, as was shown in [39]. The above BPS equations also clarify why the two-dimensional chiral equations in Section 4.1 reappear in Eq.(4.23).

\section{3 $D=8$ and $D=10$}

The analysis for the first-order system (2.19) becomes much more complicated in higher dimensions. The unbroken supersymmetries in $D=8$ have been analyzed in [23]. Because the integrable structure of Einstein equations in higher dimensions is little known, it is difficult to precisely identify governing geometrical structures emergent from the gauge theory (2.2) or (2.13) even for BPS states. Nevertheless some BPS configurations can be easily implemented as follows. As we did in Eqs.(4.24)-(4.25), one can imbed the 4-dimensional self-dual system for $n=1$ or $n=2$ into eight or ten dimensions. The simplest case is that the metric (3.4) becomes (locally) a product manifold $\mathcal{M}_{4} \times X$ where $\mathcal{M}_{4}$ is a self-dual (hyper-Kähler) four-manifold. For example, we can consider an eight-dimensional configuration where $\left(A_{1}, A_{2}, \Phi^{3}, \Phi^{4}\right)$ depend only on $\left(z^{1}, z^{2}, y^{3}, y^{4}\right)$ coordinates while $\left(\Phi^{1}, \Phi^{2}, A_{3}, A_{4}\right)$ do only on $\left(y^{1}, y^{2}, z^{3}, z^{4}\right)$ in a $B$-field background with $\theta^{12} \neq 0$ and $\theta^{34} \neq 0$, only non-vanishing components. There are many similar configurations. We will not exhaust them, instead we will consider the simplest cases which already have some relevance to other works.

The simplest BPS state in $D=8$ is the case with $n=2$ in the action (2.13); see Eq.(55) in [23]. 
The equations are of the form

$$
\begin{aligned}
& F_{\mu \nu}= \pm \frac{1}{2} \varepsilon_{\mu \nu \lambda \sigma} F_{\lambda \sigma}, \\
& {\left[\Phi^{a}, \Phi^{b}\right]= \pm \frac{1}{2} \varepsilon_{a b c d}\left[\Phi^{c}, \Phi^{d}\right],} \\
& D_{\mu} \Phi^{a}=0 .
\end{aligned}
$$

A solution of Eq.(4.28) is given by $A_{\mu}=A_{\mu}(z)$ and $\Phi^{a}=\Phi^{a}(y)$. Then Eq.(4.26) becomes commutative Abelian equations which allow no non-singular solutions, while (4.27) reduces to Eq.(3.5) describing 4-dimensional self-dual manifolds [15]. Thus the metric (3.4) in this case leads to a halfBPS geometry $\mathbb{R}^{4} \times \mathcal{M}_{4}$. Since we don't need instanton solutions in Eq.(4.26), we may freely replace $\mathbb{R}^{4}$ by 4-dimensional Minkowski space $\mathbf{R}^{1,3}$ (see the footnote 1 ).

The above system was considered in [40] in the context of D3-D7 brane inflationary model. The model consists of a D3-brane parallel to a D7-brane at some distance in the presence of $\mathcal{F}_{a b}=$ $(B+F)_{a b}$ on the worldvolume of the D7-brane, but transverse to the D3-brane. The $\mathcal{F}$-field plays the role of the Fayet-Illiopoulos term from the viewpoint of the D3-brane worldvolume field theory. Because of spontaneously broken supersymmetry in de Sitter valley the D3-brane is attracted towards the D7-brane and eventually it is dissolved into the D7-brane as a NC instanton. The system ends in a supersymmetric Higgs phase with a smooth instanton moduli space. An interesting point in [40] is that there is a relation between cosmological constant in spacetime and noncommutativity in internal space. Our above result adds a geometrical picture that the internal space after tachyon condensation is developed to a gravitational instanton, e.g., an ALE space or $K 3$.

Another interesting point, not mentioned in [40], is that it effectively realizes the dynamical compactification of extra dimensions suggested in [41]. Since the D3-brane is an instanton inside the D7-brane, particles living in the D3-brane are trapped in the core of the instanton with size $\sim \theta^{2}$ where the noncommutativity scale $\theta$ is believed to be roughly Planck scale. Since the instanton (D3-brane) results in a spontaneous breaking of translation symmetry and supersymmetry partially, Goldstone excitations corresponding to the broken bosonic and fermionic generators are zero-modes trapped in the core of the instanton. "Quarks" and "leptons" might be identified with these fermionic zero-modes [41].

We argued in the last of Section 3 that the 10-dimensional metric (3.4) for $d=4$ and $n=3$ reasonably corresponds to an emergent geometry arising from the 4-dimensional $\mathcal{N}=4$ supersymmetric $U(N)$ Yang-Mills theory. Especially it may be closely related to the bubbling geometry in AdS space found by Lin, Lunin and Maldacena (LLM) [18]. One may notice that the LLM geometry is a bubbling geometry deformed from the $A d S_{5} \times \mathbf{S}^{5}$ background which can be regarded as a vacuum manifold emerging from the self-dual RR five-form background, while the Ward's geometry (3.4) is defined in a 2-form $B$-field background and becomes (conformally) flat if all fluctuations are turned off, say, $\left(A_{\mu}, \Phi^{a}\right) \rightarrow\left(0, y^{a} / \kappa\right)$. But it turns out that the LLM geometry is a special case of the Ward's geometry (3.4). 
To see this, recall that the $A d S_{5} \times \mathbf{S}^{5}$ background is conformally flat, i.e.,

$$
d s^{2}=\frac{L^{2}}{\rho^{2}}\left(\eta_{\mu \nu} d z^{\mu} d z^{\nu}+d y^{a} d y^{a}\right)=\frac{L^{2}}{\rho^{2}}\left(\eta_{\mu \nu} d z^{\mu} d z^{\nu}+d \rho^{2}\right)+L^{2} d \Omega_{5}^{2}
$$

where $\rho^{2}=\sum_{a=1}^{6} y^{a} y^{a}$ and $d \Omega_{5}^{2}$ is the spherically symmetric metric on $\mathbf{S}^{5}$. It is then easy to see that the metric (4.29) is exactly the vacuum geometry of Eq.(3.4) when the volume form $\omega$ in Eq.(3.2) is given by

$$
\omega=\frac{d y^{1} \wedge \cdots \wedge d y^{6}}{\rho^{2}}
$$

Therefore it is obvious that the Ward's metric (3.4) with the volume form (4.30) describes a bubbling geometry which approaches to the $A d S_{5} \times \mathbf{S}^{5}$ space at infinity where fluctuations are vanishing, namely, $\left(A_{\mu}, \Phi^{a}\right) \rightarrow\left(0, y^{a} / \kappa\right)$. Note that the flat spacetime $\mathbf{R}^{1,9}$ is coming from the volume form $\omega=d y^{1} \wedge \cdots \wedge d y^{6}$, so Eq. (4.30) should correspond to some nontrivial soliton background from the gauge theory point of view. We will discuss in Section 5 a possible origin of the volume form (4.30).

Now let us briefly summarize half-BPS geometries of type IIB string theory corresponding to the chiral primaries of $\mathcal{N}=4$ super Yang-Mills theory [18]. These BPS states are giant graviton branes which wrap an $\mathbf{S}^{3}$ in $A d S_{5}$ or an $\widetilde{\mathbf{S}}^{3}$ in $\mathbf{S}^{5}$. Thus the geometry induced (or back-reacted) by the giant gravitons preserves $S O(4) \times S O(4) \times R$ isometry. It turns out that the solution is completely determined by a single function which is specified with two types of boundary conditions on a particular plane corresponding to either of two different spheres shrinking on the plane in a smooth fashion. The LLM solutions are thus in one-to-one correspondence with various 2-colorings of a 2-plane, usually referred to as 'droplets' and the geometry depends on the shape of the droplets. The droplet describing gravity solutions turns out to be the same droplet in the phase space describing free fermions for the half-BPS states.

The solutions can be analytically continued to those with $S O(2,2) \times S O(4) \times U(1)$ symmetry [18], so the solutions have the $A d S_{3} \times \mathbf{S}^{3}$ factor rather than $\mathbf{S}^{3} \times \widetilde{\mathbf{S}}^{3}$. After an analytic continuation, a underlying 4-dimensional geometry $\mathcal{M}_{4}$ attains a nice geometrical structure at asymptotic region, where $A d S_{3} \times \mathbf{S}^{3} \rightarrow \mathbb{R}^{1,5}$ and $\mathcal{M}_{4}$ reduces to a hyper-Kähler geometry. But it loses the nice picture in terms of fermion droplet since the solution is now specified by one type of boundary condition. It is interesting to notice that the asymptotic bubbling geometry for the type IIB case is the GibbonsHawking metric [28] and the real heaven metric [42] for the $M$ theory case, which are all solutions of NC electromagnetism [15, 16].

It is quite demanding to completely determine general half-BPS geometries emerging from the gauge-Higgs system in the action (2.13). Hence we will look at only an asymptotic geometry (or a local geometry) which is relatively easy to identify. For the purpose, we consider the $n=3$ case on 4-dimensional Minkowski space $\mathbb{R}^{1,3}$. It is simple to mimic the previous half-BPS configurations in $D=6,8$ with trivial extra Higgs fields. Then the resulting metric (3.4) will be locally of the form $\mathcal{M}_{4} \times \mathbf{R}^{1,5}$ akin to the asymptotic bubbling geometry. However $\mathcal{M}_{4}$ can be a general hyper-Kähler manifold. Therefore the solutions we get will be more general, whose explicit form will depend on 
underlying Killing symmetries and boundary conditions. For example, the type IIB case is given by a hyper-Kähler geometry with one translational Killing vector (Gibbons-Hawking) while the M theory case is with one rotational Killing vector (real heaven) [43]. Therefore we may get in general bubbling geometries in the $\mathrm{M}$ theory as well as the type IIB string theory.

\section{Discussion}

We showed reasonable evidences that the 10-dimensional metric (3.4) for $d=4$ and $n=3$ describes the emergent geometry arising from the 4-dimensional $\mathcal{N}=4$ supersymmetric $U(N)$ Yang-Mills theory and thus might explain the AdS/CFT duality [12]. An important point in this context is that the volume form (4.30) is required to describe the $A d S_{5} \times \mathbf{S}^{5}$ background. What is the origin of this nontrivial volume form ? In other words, how to realize the self-dual RR five-form background from the gauge theory point of view?

To get some hint about the question, first note that the $A d S_{5} \times \mathbf{S}^{5}$ geometry emerges from multiinstanton collective coordinates which dominates the path integral in a large $N$ limit [44]. The factor $d^{4} z d \rho \rho^{-5}$ appears in bosonic collective coordinate integration (with $z^{\mu}$ the instanton 4-positions) which agrees with the volume form of the conformally invariant space $A d S_{5}$, where instanton size corresponds to the radial coordinate $\rho$ in Eq.(4.29). Another point is that the $A d S_{5} \times \mathbf{S}^{5}$ space corresponds to the LLM geometry for the simplest and most symmetric configuration which reduces to the usual Gibbons-Hakwing metric (3.6) at asymptotic regions [18]. This result is consistent with the picture in Section 4.2 that $U(N)$ instantons at large $N$ limit are indeed gravitational instantons. It is then tempted to speculate that the $A d S_{5} \times \mathbf{S}^{5}$ geometry would be emerging from a maximally supersymmetric instanton solution of Eq.(2.19) in $D=10$. It should be an interesting future work.

In addition, we would like to point out that an $A d S_{p} \times \mathbf{S}^{q}$ background arises from Eq. (3.4) in the same way as Eq.(4.30) by choosing the volume form $\omega$ as follows

$$
\omega=\frac{d y^{1} \wedge \cdots \wedge d y^{q+1}}{\rho^{2}}
$$

with $\rho^{2}=\sum_{a=1}^{q+1} y^{a} y^{a}$ and $\left(A_{\mu}, \Phi^{a}\right)=\left(0, y^{a} / \kappa\right)$. A particularly interesting case is $d=2$ and $n=2$ for which the volume form (5.1) leads to the $A d S_{3} \times \mathrm{S}^{3}$ background and the action (2.13) describes matrix strings [45, 46]. We believe that the metric (3.4) with $\omega=d y^{1} \wedge \cdots \wedge d y^{4} / \rho^{2}$ describes a bubbling geometry emerging from the matrix strings.

One might already notice a subtle difference between the matrix action (2.13) and the Ward's metric (3.4). According to our construction in Section 2, the number of the Higgs fields $\Phi^{a}$ is even while the Ward construction has no such restriction. But it was shown in [15, 16] that the GibbonsHawking metric (3.6) for the $d=3$ and $m=1$ case also arises from the $d=0$ and $m=4$. It implies that we can replace some transverse scalars by gauge fields and vice versa. Recalling that the fields in the action (2.13) are all $N \times N$ matrices, of course, $N \rightarrow \infty$, it is precisely 'matrix $T$-duality' 
exchanging transverse scalars and gauge fields associated with a compact direction in $p$-brane and $(p+1)$-brane worldvolume theories through (see Eq.(154) in [46])

$$
\Phi^{a} \leftrightarrow i D_{\mu}=i\left(\partial_{\mu}-i A_{\mu}\right)
$$

With this identification, the $d$-dimensional $U(N)$ gauge theory 2.13 can be obtained by applying the $d$-fold 'matrix $T$-duality' (5.2) to the 0 -dimensional IKKT matrix model [11, 20]

$$
S=-\frac{2 \pi \kappa^{2}}{g_{s}} \operatorname{Tr}\left(\frac{1}{4} g_{M P} g_{N Q}\left[\Phi^{M}, \Phi^{N}\right]\left[\Phi^{P}, \Phi^{Q}\right]\right) .
$$

However, the $T$-duality (5.2) gives rise to qualitatively radical changes in worldvolume theory. First it changes the dimensionality of the theory and thus it affects its renormalizability (see Sec. VI in [46] and references therein for this issue in Matrix theory). For example, the action (2.13) for $d>4$ is not renormalizable since the coupling constant $g_{Y M}^{2} \sim g_{s} \kappa^{\frac{d-4}{2}} \sim g_{s} m_{s}^{4-d}$ has negative mass dimension in this case. Second it also changes a behavior of the emergent metric (3.4). But these changes are rather consistent with the fact that under the $T$-duality (5.2) a $\mathrm{D} p$-brane is transformed into a $\mathrm{D}(p+1)$-brane and vice versa.

Our construction in Section 2 raises a bizarre question about the renormalization property of NC field theory. If we look at the action (2.2), the theory superficially seems to be non-renormalizable for $D>4$ since the coupling constant (2.5) has negative mass dimension. But this non-renormalizability appears as a fake if we use the matrix representation (2.18) together with the redefinition of variables in Eq.(2.4). The resulting coupling constant, denoted as $g_{d}$, in the final action (2.13) depends only on the dimension of commutative spacetime rather than the entire spacetime. Since the resulting $U(N)$ theory is in the limit $N \rightarrow \infty$, while the 't Hooft coupling $\lambda \equiv g_{d}^{2} N$ is kept fixed, planar diagrams dominate in this limit [9]. Since the dependence of NC coordinates in the action (2.2) has been encoded into the matrix degrees of freedom, one may suspect that the divergence of the original theory might appear as a divergence of perturbation series as a whole in the action (2.13). The convergence aspect of the planar perturbation theory concerns $N_{p}(n)$, the number of planar diagrams in $n$th order in $\lambda$. It was shown in [47] that $N_{p}(n)$ behaves asymptotically as

$$
N_{p}(n) \stackrel{n \rightarrow \infty}{\sim} c^{n}, \quad c=\text { constant. }
$$

Therefore the planar theory (unlike the full theory) for $d \leq 4$ has a formally convergent perturbation series, provided the ultraviolet and infrared divergences of individual diagrams are cut off [47]. It will be interesting to carefully examine the renormalization property of NC field theories along this line.

We showed in Section 3 that the Ward metric (3.4) is emerging from commutative, i.e., $\mathcal{O}(\theta)$, limit. Since the vector fields in Eq.(3.11) are in general higher order differential operators acting on $\mathcal{A}_{\theta}$, we thus expect that they actually define a 'generalized gravity' beyond Einstein gravity, e.g., the NC gravity [29] or the NC unimodular gravity [48] 6] It was shown in [16] that the leading derivative

\footnotetext{
${ }^{6}$ The latter seems to be quite relevant to our emergent gravity since the vector fields $V_{M}$ in Eq. (3.11) always belong to the volume preserving diffeomorphisms, which is a generic property of vector fields defined in NC spacetime. It should be interesting to more clarify the relation between the NC unimodular gravity [48] and the emergent gravity.
} 
corrections in NC gauge theory start with four derivatives, which was conjectured to give rise to higher order gravity. As was explicitly checked for the self-dual case, Einstein gravity maybe emerges from NC gauge fields in commutative limit, which then implies that the leading derivative corrections give rise to higher order terms with four more derivatives compared to the Einstein gravity. This means that the higher order gravity starts from the second order corrections in $\theta$ with higher derivatives, that is, no first order correction in $\theta$ to the Einstein gravity. Interestingly this result is consistent with those in [29] and also in [49] calculated from the context of NC gravity.

It was shown in Section 4.1 that the self-duality system for the $D=4$ and $n=1$ case is mapped to the two-dimensional $U(\infty)$ chiral model (4.9) which is remarkably equivalent to self-dual Einstein gravity [33, 17, 34]. But this case should not be much different from the $D=4$ and $n=2$ case in [15, 16] since they equally describe the self-dual Einstein gravity. Indeed we can make them bear a close resemblance each other. For the purpose, let us consider a four-dimensional NC space $\mathbb{R}_{N C}^{2} \times \mathbb{R}_{N C}^{2}$. We can choose the matrix representation (2.18) only for the second factor, i.e.,

$$
\phi\left(y^{1}, y^{2}, y^{3}, y^{4}\right)=\sum_{i, j} \Omega_{i j}\left(y^{1}, y^{2}\right)|i\rangle\langle j|
$$

As a result, the action (2.13) now becomes two-dimensional $U(N)$ gauge theory on $\mathbb{R}_{N C}^{2}$. The selfdual equations in Eq. (4.1) in this case are given by the NC Hitchin equations, now defined on $\mathbf{R}_{N C}^{2}$ instead of $\mathbf{R}_{C}^{2}$. The NC Hitchin equations have been considered by K. Lee in [37] with very parallel results with the commutative case (4.1). It is interesting that there exist two different realizations for self-dual Einstein gravity, whose relationship should be more closely understood.

Finally it will be interesting to consider a compact NC space instead of $\mathbf{R}_{N C}^{2 n}$, for instance, a NC $2 n$-torus $\mathbf{T}_{N C}^{2 n}$. Since the module over a NC torus is still infinite dimensional [8], the matrix representation (2.18) is also infinite dimensional. Thus we expect that our construction in Section 2 and 3 can be applied even to the NC torus without many essential changes.

\section{Acknowledgments}

After posting this paper to the arXiv, we were informed of related works on YM-Higgs BPS configurations on NC spaces [50] and on the relation between a large $N$ gauge theory, a Moyal deformation and a self-dual gravity [51] by O. Lechtenfeld and C. Castro, respectively. We thank them for the references. This work was supported by the Alexander von Humboldt Foundation. 


\section{References}

[1] M. R. Douglas and N. A. Nekrasov, Noncommutative field theory, Rev. Mod. Phys. 73, 977 (2001), hep-th/0106048; R. J. Szabo, Quantum field theory on noncommutative spaces, Phys. Rep. 378, 207 (2003), hep-th/0109162.

[2] R. J. Szabo, Symmetry, gravity and noncommutativity, Class. Quantum Grav. 23, R199 (2006), hep-th/0606233.

[3] H. S. Yang, On The Correspondence Between Noncommuative Field Theory And Gravity, Mod. Phys. Lett. A22, 1119 (2007), hep-th/0612231.

[4] M. Kontsevich, Deformation Quantization of Poisson Manifolds, Lett. Math. Phys. 66, 157 (2003), q-alg/9709040.

[5] J. Madore, The fuzzy sphere, Class. Quantum Grav. 9, 69 (1992).

[6] J. A. Harvey, Topology of the Gauge Group in Noncommutative Gauge Theory, hep-th/0105242.

[7] F. Lizzi, R. J. Szabo and A. Zampini, Geometry of the gauge algebra in noncommutative YangMills theory, J. High Energy Phys. 08, 032 (2001), hep-th/0107115.

[8] N. Seiberg and E. Witten, String theory and noncommutative geometry, J. High Energy Phys. 09, 032 (1999), hep-th/9908142.

[9] G. 't Hooft, A planar diagram theory for strong interactions, Nucl. Phys. B72, 461 (1974).

[10] T. Banks, W. Fischler, S. H. Shenker and L. Susskind, $M$ theory as a matrix model: A conjecture, Phys. Rev. D55, 5112 (1997), hep-th/9610043.

[11] N. Ishibashi, H. Kawai, Y. Kitazawa and A. Tsuchiya, A large-N reduced model as superstring, Nucl. Phys. B498, 467 (1997), hep-th/9612115.

[12] J. M. Maldacena, The Large N Limit of Superconformal Field Theories and Supergravity, Adv. Theor. Math. Phys. 2, 231 (1998); Int. J. Theor. Phys. 38, 1113 (1999), hep-th/9711200; S. S. Gubser, I. R. Klebanov and A. M. Polyakov, Gauge Theory Correlators from Non-Critical String Theory, Phys. Lett. B428, 105 (1998), hep-th/9802109; E. Witten, Anti De Sitter Space And Holography, Adv. Theor. Math. Phys. 2, 253 (1998), hep-th/9802150.

[13] M. Salizzoni, A. Torrielli and H. S. Yang, ALE spaces from noncommutative U(1) instantons via exact Seiberg-Witten map, Phys. Lett. B634, 427 (2006), hep-th/0510249.

[14] H. S. Yang and M. Salizzoni, Gravitational Instantons from Gauge Theory, Phys. Rev. Lett. 96, 201602 (2006), hep-th/0512215. 
[15] H. S. Yang, Instantons and Emergent Geometry, hep-th/0608013.

[16] H. S. Yang, Emergent Gravity from Noncommutative Spacetime, hep-th/0611174.

[17] R. S. Ward, The $S U(\infty)$ chiral model and self-dual vacuum spaces, Class. Quantum Grav. 7, L217 (1990).

[18] H. Lin, O. Lunin and J. Maldacena, Bubbling AdS space and 1/2 BPS geometries, J. High Energy Phys. 10, 025 (2004), hep-th/ 0409174 ; H. Lin and J. Maldacena, Fivebranes from gauge theory, Phys. Rev. D74, 084014 (2006), hep-th/ 0509235.

[19] N. Seiberg, A note on background independence in noncommutative gauge theories, matrix model and tachyon condensation, J. High Energy Phys. 09, 003 (2000), hep-th/ 0008013.

[20] H. Aoki, N. Ishibashi, S. Iso, H. Kawai, Y. Kitazawa and T. Tada, Non-commutative Yang-Mills in IIB matrix model, Nucl. Phys. B565 (2000) 176, hep-th/9908141.

[21] E. Corrigan, C. Devchand, D. B. Fairlie and J. Nuyts, First-order equations for gauge fields in spaces of dimension greater than four, Nucl. Phys. B214, 452 (1983).

[22] R. S. Ward, Completely solvable gauge-field equations in dimension greater than four, Nucl. Phys. B236, 381 (1984).

[23] D. Bak, K. Lee and J.-H. Park, BPS equations in six and eight dimensions, Phys. Rev. D66, 025021 (2002), hep-th/0204221.

[24] A. Ashtekar, T. Jabobson and L. Smolin, A New Characterization Of Half-Flat Solutions to Einstein's Equation, Commun. Math. Phys. 115, 631 (1988).

[25] L. J. Mason and E. T. Newman, A Connection Between the Einstein and Yang-Mills Equations, Commun. Math. Phys. 121, 659 (1989).

[26] S. Chakravarty, L. Mason and E. T. Newman, Canonical structures on anti-self-dual fourmanifolds and the diffeomorphism group, J. Math. Phys. 32, 1458 (1991).

[27] D. D. Joyce, Explicit Construction of Self-dual 4-Manifolds, Duke Math. J. 77, 519 (1995).

[28] G. W. Gibbons and S. W. Hawking, Gravitational Multi-instantons, Phys. Lett. 78B, 430 (1978).

[29] P. Aschieri, C. Blohmann, M. Dimitrijevic, F. Meyer, P. Schupp and J. Wess, A gravity theory on noncommutative spaces, Class. Quant. Grav. 22, 3511 (2005), hep-th/0504183; P. Aschieri, M. Dimitrijevic, F. Meyer and J. Wess, Noncommutative geometry and gravity, Class. Quant. Grav. 23, 1883 (2006), hep-th/0510059. 
[30] R. Gopakumar and D. J. Gross, Mastering the master field, Nucl. Phys. B451, 379 (1995), hep-th/9411021; I. Ya. Aref'eva and I. V. Volovich, The master field for QCD and $q$ deformed quantum field theory, Nucl. Phys. B462, 600 (1996), hep-th/9510210.

[31] J. Madore, S. Schraml, P. Schupp and J. Wess, Gauge theory on noncommutative spaces, Eur. Phys. J. C16, 161 (2000), hep-th/0001203.

[32] C.-S. Chu, V. V. Khoze and G. Travaglini, Notes on noncommutative instantons, Nucl. Phys. B621, 101 (2002), hep-th/0108007; K.-Y. Kim, B.-H. Lee and H. S. Yang, Noncommutative instantons on $\mathbf{R}_{N C}^{2} \times \mathbf{R}_{C}^{2}$, Phys. Lett. B523, 357 (2001), hep-th/0109121.

[33] Q-H. Park, Self-dual Gravity as A Large-N Limit of the 2D Non-linear Sigma Model, Phys. Lett. B238, 287 (1990).

[34] V. Husain, Self-Dual Gravity and the Chiral Model, Phys. Rev. Lett. 72, 800 (1994), gr-qc/9402020.

[35] G. V. Dunne, R. Jackiw, S.-Y. Pi and C. A. Trugenberger, Self-dual Chern-Simons solitons and two-dimensional nonlinear equations, Phys. Rev. D43, 1332 (1991); Erratum-ibid. D45, 3012 (1992).

[36] G. V. Dunne, Chern-Simons solitons, Toda theories and the chiral model, Commun. Math. Phys. 150, 519 (1992), hep-th/9204056.

[37] K.-M. Lee, Chern-Simons solitons, chiral model, and (affine) Toda model on noncommutative space, J. High Energy Phys. 08, 054 (2004), hep-th/ 0405244.

[38] J. F. Plebañski, Some solutions of complex Einstein equations, J. Math. Phys. 16, 2395 (1575).

[39] J. F. Plebański and M. Przanowski, The Lagrangian of a self-dual gravitational field as a limit of the SDYM Lagrangian, Phys. Lett. A212, 22 (1996), hep-th/9605233.

[40] K. Dasgupta, C. Herdeiro, S. Hirano and R. Kallosh, D3-D7 inflationary model and M theory, Phys. Rev. D65, 126002 (2002), hep-th/0203019.

[41] G. Dvali and M. Shifman, Dynamical compactification as a mechanism of spontaneous supersymmetry breaking, Nucl. Phys. B504, 127 (1997), hep-th/9611213.

[42] C. P. Boyer and J. D. Finley, III, Killing vectors in self-dual, Euclidean Einstein spaces, J. Math. Phys. 23, 1126 (1982).

[43] I. Bakas and K. Sfetsos, Toda Fields of $\mathrm{SO}(3)$ Hyper-Kahler Metrics and Free Field Realizations, Int. J. Mod. Phys. A12, 2585 (1997), hep-th/9604003. 
[44] M. Bianchi, M. B. Green, S. Kovacs and G. Rossi, Instantons in supersymmetric YangMills and D-instantons in IIB superstring theory, J. High Energy Phys. 08, 013 (1998), hep-th/9807033; N. Dorey, T. J. Hollowood, V. V. Khoze, M. P. Mattis and S. Vandoren, Multi-instanton calculus and the AdS/CFT correspondence in $\mathcal{N}=4$ superconformal field theory, Nucl. Phys. B552, 88 (1999), hep-th/9901128.

[45] L. Motl, Proposals on nonperturbative superstring interactions, hep-th/9701025; R. Dijkgraaf, E. Verlinde and H. Verlinde, Matrix string theory, Nucl. Phys. B500, 43(1997), hep-th/9703030.

[46] W. Taylor, M(atrix) theory: matrix quantum mechanics as a fundamental theory, Rev. Mod. Phys. 73, 419 (2001), hep-th/0101126.

[47] J. Koplik, A. Neveu and S. Nussinov, Some aspects of the planar perturbation series, Nucl. Phys. B123, 109 (1977).

[48] X. Calmet and A. Kobakhidze, Noncommutative general relativity, Phys. Rev. D72, 045010 (2005), hep-th/0506157.

[49] P. Mukherjee and A. Saha, Note on the noncommutative correction to gravity, Phys. Rev. D74, 027702 (2006), hep-th/ 0605287 ; X. Calmet and A. Kobakhidze, Second order noncommutative corrections to gravity, Phys. Rev. D74, 047702 (2006), hep-th/ 0605275 ; R. Banerjee, P. Mukherjee and S. Samanta, Lie algebraic Noncommutative Gravity, Phys. Rev. D75, 125020 (2007), hep-th/0703128.

[50] O. Lechtenfeld, A. D. Popov and R. J. Szabo, Noncommutative instantons in higher dimensions, vortices and topological K-cycles, J. High Energy Phys. 12, 022 (2003), hep-th/0310267; A. V. Domrin, O. Lechtenfeld and S. Petersen, Sigma-Model Solitons in the Noncommutative Plane: Construction and Stability Analysis, J. High Energy Phys. 03, 045 (2005), hep-th/0412001; O. Lechtenfeld, A. D. Popov and R. J. Szabo, Rank two quiver gauge theory, graded connections and noncommutative vortices, J. High Energy Phys. 09, 054 (2006), hep-th/0603232.

[51] C. Castro, $S U(\infty)$ (super)gauge theories and self-dual (super)gravity, J. Math. Phys. 34, 681 (1993); The $N=2$ super-Wess-Zumino-Novikov-Witten model valued in superdiffeomorphism (SDIFF) $M^{2}$ is self-dual supergravity in four dimensions, J. Math. Phys. 35, 920 (1994); C. Castro and J. Plebański, The generalized Moyal-Nahm and continuous Moyal-Toda equations, J. Math. Phys. 40, 3738 (1996), hep-th/9710041; C. Castro, A Moyal quantization of the continuous Toda field, Phys. Lett. B413, 53 (1997), hep-th/9703094; S. Ansoldi, C. Castro and E. Spallucci, Chern-Simons hadronic bag from quenched large- $N$ QCD, Phys. Lett. B504, 174 (2001), hep-th/0011013. 